\title{
On the Number of Solutions of Certain Trinomial Congruences
}

\section{By Jacqueline Wells and Joseph Muskat}

1. In the course of his extensive investigations into Fermat's Last Theorem, H. S. Vandiver considered the number of solutions $(x, y)$ of

$$
1+a x^{c} \equiv b y^{e} \quad(\bmod p),
$$

where $c e+1=p$, a prime, and $x y \not \equiv 0(\bmod p)$.

If $a b \not \equiv 0(\bmod p)$, the following is an equivalent formulation: Let $g$ be a primitive root of $p$. Then determine, for $i$ and $j$ fixed, $0 \leqq i \leqq c-1,0 \leqq j \leqq e-1$, the number of pairs $(r, s), 0 \leqq r \leqq e-1,0 \leqq s \leqq c-1$, for which the congruence

$$
1+g^{i+c r} \equiv g^{j+e s} \quad(\bmod p)
$$

is solvable. The number of solutions $(r, s)$ will be denoted by $[i, j]_{c e}$, or simply by $[i, j]$, if $c$ and $e$ are fixed.

$$
\begin{array}{ll}
e-1, & e \text { even, } \quad i=0, \\
\sum_{j=0}^{e-1}[i, j]_{c e}=\begin{array}{l}
e \\
e-1,
\end{array} & e \text { odd, } i=c / 2, \\
e, & \text { otherwise }
\end{array}
$$

It follows from $(2)$ that $[i, j]_{c e} \leqq e$.

For a fixed $e$ and $p$, let $N_{k}$ denote the number of the $c e$-pairs $(i, j)$ for which $[i, j]_{c e}=k$.

Given a fixed $i$ for which

$$
\sum_{j=0}^{e-1}[i, j]_{c e}=e
$$

then the integers $[i, 0],[i, 1],[i, 2], \cdots,[i, e-2],[i, e-1]$ form a partition of $e$. The partition $e 00 \cdots 00$, where, for a fixed $i$, there is one $j$ such that $[i, j]=e$, and, for all other $j,[i, j]=0$, enters into a criterion for Fermat's Last Theorem [3, Theorem 2].

Erna H. Pearson computed the values of the $[i, j]$ for several values of $e$ and $p$. A list of the cases she considered can be found in [2, p. 1284]. During 1954 and 1955, Emma Lehmer, J. L. Selfridge, and C. A. Nicol, with the aid of the swac digital computer, computed the values of $[i, j]$ for $e=5,7, p<1024 ; e=11, p<800$; $e=13, p<600$; and $e$ prime, $17 \leqq e<256, p<512$. For each $p$, the $N_{k}, k=0$, $1, \cdots, e$, were determined, and the occurrences of each of the partitions of $e$ were tallied [1].

In [1], it was suggested that the values of the $N_{k}$ and the occurrences of the various partitions deviated significantly from what was "expected." To probe this situation, we undertook to calculate the $[i, j]$, the values of the $N_{k}$, and the occurrences of the various partitions for $e=5,7,9,11$, and 13, $p<18,000$. We leave to the end a brief description of the program.

Received September 25, 1964. This research was sponsored in part by the National Science Foundation, under Research Grants G11309 and GP-2091. 
2. We assumed the following probability model: Given $e$ objects, each is to be put independently into one of $e$ cells. (We neglect the fact that ours is a sampling without replacement situation.) Then the probability that a given cell contains exactly $k$ objects is given by the binomial distribution as

$$
\left(\begin{array}{l}
e \\
k
\end{array}\right)\left(\frac{1}{e}\right)^{k}\left(\frac{e-1}{e}\right)^{e-k} \text {. }
$$

In the problem considered here, the objects are the $e$ solutions of (1) with $i$ fixed. The cells are the values $0,1, \cdots, e-1$ which $j$ may take.

Table 1 shows, for $e=5$, the expected and the observed occurrences of the $N_{k}$, $k=0,1, \cdots, 5$.

For each value of $e$ in the study, the primes $p \equiv 1(\bmod e)$, arranged in ascending order, were subdivided into several groups. Within each group, the values of $N_{k} /(p-1)$, for each $k, 0 \leqq k \leqq e$, were sorted from low to high and every $n$th value, where $n$ depended upon $e$, was recorded. For $e=5$, the first six primes were omitted and the remaining 505 in the study were divided into five groups of 101 each. In Table 2 , every $n$th value of $N_{0} /(p-1), n=1,21,41,61,81,101$, is recorded for each of the five groups.

Tables 1 and 2, being typical of the tables generated in this study, suggest that this probability model approximates the actual situation quite well. Table 2 and similar tables indicate that, as $p$ increases, the approximation improves. The full set of tables can be found in [4].

3. In [1] the occurrences of the various partitions of $e$ among the solutions of (1) for fixed $i$ were tabulated. According to the probability model outlined in Section 2,

TABLE 1

\begin{tabular}{cc|c|c|c}
\hline & $\begin{array}{c}\text { Proportion } \\
\text { expected }\end{array}$ & $\begin{array}{c}\text { Proportion } \\
\text { observed }\end{array}$ & Occurrences \\
\hline & $N_{0}$ & .32768 & .32761 & $1,419,388$ \\
& $N_{1}$ & .40960 & .40979 & $1,775,443$ \\
& $N_{2}$ & .20480 & .20472 & 886,952 \\
& $N_{3}$ & .05120 & .05115 & 221,602 \\
& $N_{4}$ & .00640 & .00642 & 27,818 \\
& $N_{\mathrm{b}}$ & .00032 & .00030 & 1,317 \\
\hline Totals & 1.00000 & .99999 & $4,332,520$ \\
\hline
\end{tabular}

TABLE 2

Distribution of $N_{0} /(p-1), e=5$, Within Groups

\begin{tabular}{|c|c|c|c|c|c|c|c|}
\hline Group & Range of $p$ & $1 \mathrm{st}$ & 21 st & 41st & $61 \mathrm{st}$ & 81 st & $101 \mathrm{st}$ \\
\hline 1 & $131-3061$ & .31391 & .32432 & .32660 & .32791 & .32983 & .33906 \\
\hline 2 & $3121-6451$ & .31974 & .32588 & .32696 & .32800 & .32919 & .33534 \\
\hline$\overline{3}$ & $6481-10111$ & .32460 & .32597 & .32719 & .32805 & .32881 & .33463 \\
\hline 4 & $10141-14221$ & .32434 & .32652 & .32733 & .32783 & .32883 & .33055 \\
\hline 5 & $14251-17981$ & .32522 & .32688 & .32744 & .32796 & .32852 & .33126 \\
\hline
\end{tabular}


the number of ways of obtaining a particular partition can be calculated as the product of the number $P_{1}$ of permutations of the numbers in the partition times the number $P_{2}$ of permutations of the values of $j$ in one of the $P_{1}$ permutations of the partition.

$$
P_{1}=e ! / t_{0} ! t_{1} ! t_{2} ! \cdots t_{e} !,
$$

where $t_{n}$ is the number of occurrences of $n$ in the partition.

Let $s_{j}$ denote the number of $j$ 's in a particular permutation. Then

$$
P_{2}=e ! / s_{0} ! s_{1} ! s_{2} ! \cdots s_{e-1} ! .
$$

Since the probability model has $e^{\bullet}$ equally likely outcomes, the probability of a given permutation is given by

$$
P_{1} P_{2} / e^{e} \text {. }
$$

As an illustration, consider, for $e=5$, the partition 31100 . This means that, for a fixed value of $i, P_{1}$ expresses the number of arrangements of the $j$ 's so that there is one $j$ for which $[i, j]=3$, there are two $j$ 's for which $[i, j]=1$, and for the other two $j$ 's, $[i, j]=0$.

$$
P_{1}=5 ! / 2 ! \cdot 2 ! \cdot 0 ! \cdot 1 ! \cdot 0 !=30 .
$$

One of these thirty arrangements is $[i, 2]=3,[i, 1]=[i, 4]=1,[i, 0]=[i, 3]=$ 0 . For this case, $P_{2}$ expresses the number of arrangements of the five solutions of (1) so that three of them correspond to $j=2$, and one each to $j=1$ and $j=4$.

$$
P_{2}=5 ! / 0 ! \cdot 1 ! \cdot 3 ! \cdot 0 ! \cdot 1 !=20 \text {. }
$$

Clearly, the value of $P_{2}$ is the same for each of the thirty arrangements. Thus, the probability that a given set of solutions forms the partition 31100 is

$$
30 \cdot 20 / 5^{5}=600 / 3125=0.192 .
$$

(Note that the denominator of $P_{2}$ can be obtained by affixing "factorial" symbols to all the numbers in the representation of the partition and forming the product.)

The observed and expected occurrences of the various partitions for $e=5$ and $e=7$ are shown in Tables 3 and 4 .

TABLE 3

Partitions, $e=\tilde{5}$

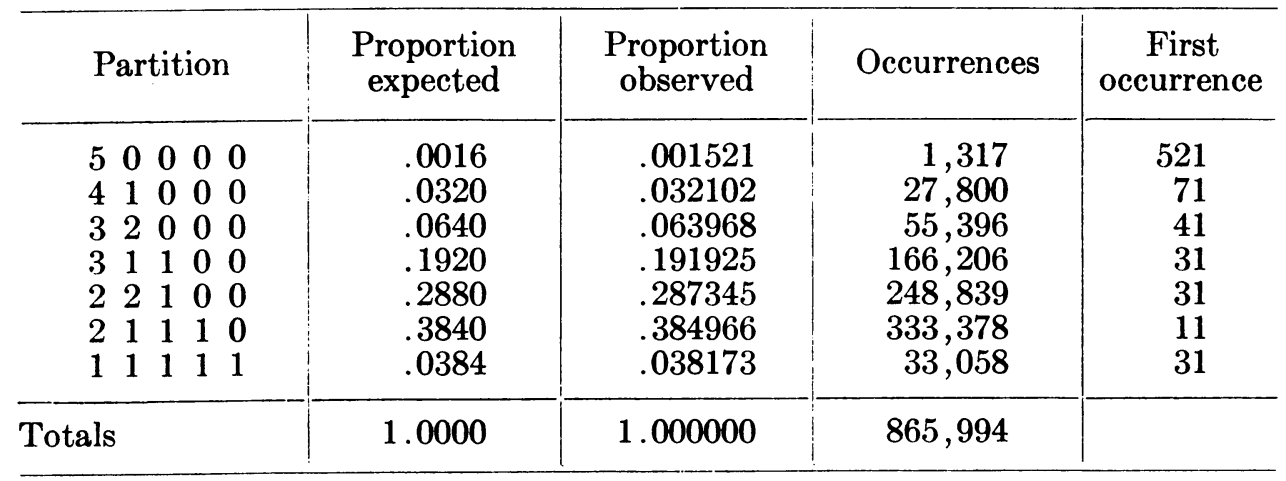


TABLE 4

Partitions, $e=7$

\begin{tabular}{|c|c|c|c|c|}
\hline Partition & $\begin{array}{c}\text { Proportion } \\
\text { expected }\end{array}$ & $\begin{array}{c}\text { Proportion } \\
\text { observed }\end{array}$ & Occurrences & $\begin{array}{c}\text { First } \\
\text { occurrence }\end{array}$ \\
\hline $\begin{array}{lllllll}7 & 0 & 0 & 0 & 0 & 0 & 0\end{array}$ & .000008 & .000007 & 3 & 9829 \\
\hline $\begin{array}{lllllll}6 & 1 & 0 & 0 & 0 & 0 & 0\end{array}$ & .000357 & .000363 & 150 & 617 \\
\hline $\begin{array}{lllllll}5 & 2 & 0 & 0 & 0 & 0 & 0\end{array}$ & .001071 & .001013 & 419 & 659 \\
\hline $\begin{array}{lllllll}5 & 1 & 1 & 0 & 0 & 0 & 0\end{array}$ & .005355 & .005349 & 2,213 & 239 \\
\hline $\begin{array}{lllllll}4 & 3 & 0 & 0 & 0 & 0 & 0\end{array}$ & .001785 & .001769 & 732 & 421 \\
\hline $\begin{array}{lllllll}4 & 2 & 1 & 0 & 0 & 0 & 0\end{array}$ & .026775 & .026445 & 10,941 & 71 \\
\hline $\begin{array}{lllllll}4 & 1 & 1 & 1 & 0 & 0 & 0\end{array}$ & .035699 & .035593 & 14,726 & 113 \\
\hline $\begin{array}{lllllll}3 & 3 & 1 & 0 & 0 & 0 & 0\end{array}$ & .017850 & .017881 & 7,398 & 127 \\
\hline 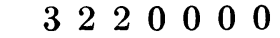 & .026775 & .026338 & 10,897 & 379 \\
\hline $\begin{array}{llllll}2 & 1 & 1 & 0 & 0 & 0\end{array}$ & .214196 & .214878 & 88,902 & 43 \\
\hline $\begin{array}{lllllll}3 & 1 & 1 & 1 & 1 & 0 & 0\end{array}$ & .107098 & .106963 & 44,254 & 29 \\
\hline 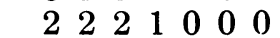 & .107098 & .107913 & 44,647 & 113 \\
\hline $\begin{array}{lllllll}2 & 2 & 1 & 1 & 1 & 0 & 0\end{array}$ & .321295 & .320731 & 132,697 & 29 \\
\hline $\begin{array}{lllllll}2 & 1 & 1 & 1 & 1 & 1 & 0\end{array}$ & .128518 & .128484 & 53,158 & 29 \\
\hline $\begin{array}{llllll}1 & 1 & 1 & 1 & 1 & 1\end{array}$ & .006120 & .006275 & 2,569 & 421 \\
\hline Totals & 1.000000 & 1.000002 & 413,733 & \\
\hline
\end{tabular}

The probability that a partition is the partition $e 00 \cdots 00$ is $1 / e^{e-1}$. The deviations mentioned at the end of Section 1 were apparently due to overlooking $P_{2}$ in computing expected occurrences.

4. The solutions of (1) were obtained on the University of Pittsburgh's IBM 7070 computer. The program differed in several respects from the program for the SWAC computer described in [1], as a much larger memory was available.

For $e=5,7,9,11$, and 13 , cards containing primes $p \equiv 1(\bmod e)$ and the least primitive root $g$ of $p$ were available from a previous study. Mr. Dale Isner of the staff of the University of Pittsburgh's Computation Center supplied a program which generated a list of the partitions of $p$.

A modified index table was generated as follows:

For each $p, g, g^{2}, g^{3}, \cdots, g^{k}, \cdots, g^{(p-1) / 2}$, reduced modulo $p$, were generated. If $n \equiv g^{k}(\bmod p), 0<n<p$, then $k$, reduced modulo $e$, was stored in cell IND + $\min \{n, p-n\}$.

The values of $g^{i+r c}$, reduced modulo $p$, were then generated. For each $i$ having $e$ values of $g^{i+r c} \not \equiv-1(\bmod p)$, consider $m \equiv g^{i+r c}(\bmod p), 0<m<p-1$. If $m<(p-1) / 2$, the number (value of $j$ ) in cell IND $+m+1$ was found and stored in a list of solutions. If $m \geqq(p-1) / 2$, the number in cell IND $+p$ $-m-1$ was stored in the solution list, as for $e$ odd,

$$
\operatorname{ind}(m+1) \equiv \operatorname{ind}(p-m-1) \quad(\bmod e) .
$$

For each $i$, the solution list was analyzed to determine the appropriate partition, and the relevant counters were tallied.

The results of the main program have been deposited in the UMT file. For each $e, e=5,7,9,11$, and 13 , the primes $p \equiv 1(\bmod e), p<18,000$, are listed, with 
the values of $N_{k}, 0 \leqq k \leqq e$, and the number of occurrences of the various partitions of $e$ into which the solutions are grouped.

Pennsylvania State University

McKeesport, Pennsylvania

University of Pittsburgh

Pittsburgh, Pennsylvanıa

1. Emma Lehmer \& H. S. VANDiver, "On the computation of the number of solutions of certain trinomial congruences," J. Assoc. Comput. Mach., v. 4, 1957, pp. 505-510. MR 20 *428.

2. E. H. Pearson \& H.S. VANdiver, "On a new problem concerning trinomial congruences involving rational integers," Proc. Nat. Acad. Sci. U.S.A., v. 39, 1953, pp. 1278-1285. MR 15, 684 .

3. H. S. VANDIVER, "New types of trinomial congruence criteria applying to Fermat's Last Theorem," Proc. Nat. Acad. Sci. U.S.A., v. 40, 1954, pp. 248-252. MR 15, 778.

4. JacQueline Wells, Studies on the Number of Solutions of a Trinomial Congruence, M. S. Thesis, University of Pittsburgh, Pittsburgh, Pa., 1964.

\section{Tables of Values of Three Infinite Integrals}

\section{By Chih-Bing Ling and Hsien-Chueh Wu}

Sometime ago, the senior author [1] evaluated the following two integrals to five decimal places for integral values of $m$ and $p$ up to 15 and 8 , respectively.

$$
\begin{array}{ll}
I(m, p)=\frac{p^{m+1}}{2^{p}(m !)} \int_{0}^{\infty} \frac{x^{m} d x}{\sinh ^{p} x} & (m \geqq p \geqq 1) \\
J(m, p)=\frac{p^{m+1}}{2^{p}(m !)} \int_{0}^{\infty} \frac{x^{m} d x}{\cosh ^{p} x} & (m \geqq 0, p \geqq 1)
\end{array}
$$

Two particular integrals $I(m, m)$ and $I(m, m-1)$ were further evaluated by Nelson and the senior author [2] to seven decimal places for $m=1$ (1)40. Nelson also evaluated these two integrals for the same range of values of $m$ to $12 \mathrm{D}$ and $18 \mathrm{D}$, respectively, in two papers [3], [4].

In the present paper, the two preceding integrals will be evaluated to $8 \mathrm{D}$ for $m$ and $p$ up to 25 and 12, respectively. The same method of evaluation will be used. The various sums of inverse powers required in the computation were tabulated to 32D by Glaisher [5], [6], and also appear in two well-known mathematical tables [7], [8]. The results are shown in Tables 1 and 2. Table 3 shows the factor $2^{p}(m !) / p^{m+1}$, also to $8 \mathrm{D}$.

In addition, the following integral will also be evaluated to $8 \mathrm{D}$ for the same range of values of $m$ and $p$.

$$
S(m, p)=\int_{0}^{\infty} \frac{\sin ^{m} x}{x^{p}} d x \quad(m \geqq p \geqq 1) .
$$

The integers $m$ and $p$ are restricted as indicated, and $S(2 m, 1)$ is to be excluded on account of its divergence. This last integral occurs in certain branches of mathematical physics, and on that account it was thought desirable to include a table of its values.

Received January 11, 1965. 